[0212-7199 (2008) 25: 4; pp 168-172] ANALES DE MEDICINA INTERNA Copyright (C) 2008 ARAN EDICIONES, S.L.

AN. MED. INTERNA (Madrid) Vol. 25, N. $^{\circ} 4$, pp. $168-172,2008$

\title{
Características y manejo de las crisis epilépticas en urgencias y su correlación diagnóstica tras el ingreso
}

\author{
G. PIÑOL-RIPOLL, J. GÓMEZ BITRIAN ${ }^{1}$, I. DE LA PUERTA GONZÁLEZ-MIRÓ², \\ R. ROYO HERNÁNDEZ', J. A. MAURI-LLERDA
}

Servicio de Neurología. ${ }^{1}$ Servicio de Urgencias. ${ }^{2}$ Servicio de Cardiología. Hospital

Clínico. Zaragoza

\begin{abstract}
CHARACTERISTICS AND MANAGEMENT OF EPILEPTIC SEIZURES IN EMERGENCY DEPARTMENT AND DIAGNOSTIC CORRELATION AT DISCHARGE
\end{abstract}

\begin{abstract}
RESUMEN
Introducción: las crisis convulsivas suponen un porcentaje elevado de las consultas en los servicios de urgencias, pero poco es conocido en relación al manejo y derivación de estos enfermos. El objetivo fue conocer el perfil de los pacientes que acuden a urgencias con crisis epilépticas, las medidas diagnósticas y terapéuticas, destino de los pacientes tras su valoración, así como la concordancia entre el diagnóstico inicial y definitivo de estos pacientes.

Material y métodos: registramos prospectivamente todos los pacientes que acudieron al Servicio de Urgencias del Hospital Clínico de Zaragoza durante el periodo comprendido entre el 1 de noviembre del 2004 y 30 de abril del 2005 y que fueron diagnosticados de crisis comicial. Posteriormente se revisaron las historias clínicas de aquellos pacientes ingresados para determinar el diagnóstico definitivo.

Resultados: de los 54.022 pacientes atendidos a urgencias, 137 $(0,25 \%)$ fueron diagnosticados de crisis convulsiva, requiriendo ingreso hospitalario $61(44 \%)$. Las crisis de novo representaron el $60 \%$ de los pacientes ingresados. Un $12 \%$ fueron secundarios a alteraciones analíticas y un $48 \%$ de los pacientes presentaron hallazgos patológicos en la neuroimagen; la falta de cumplimiento terapéutico con bajos niveles plasmáticos de fármacos fue el principal factor precipitante en los epilépticos conocidos. Los falsos positivos representaron el 33\% de los diagnósticos de urgencias.

Conclusiones: encontramos un tercio de errores respecto al diagnóstico de urgencias, siendo la causa más frecuente de error los síncopes y los ictus. Elevado porcentaje de diagnóstico etiológico por neuroimagen así como la escasez en la realización de electroencefalogramas y estudios toxicológicos.
\end{abstract}

PALABRAS CLAVE: Diagnóstico. Epilepsia. Urgencias. Exploración neurológica.

\begin{abstract}
Background: Patients with seizures are common in the emergency department $(E D)$, yet little is known regarding the management of these patients. This study was performed to define the demographic characteristics of patients with seizure disorders in the ED patient population and to determine final disposition, diagnostic and therapeutic activities, and to evaluate the concordance between provisional and definitive diagnosis.

Material and methods: We recorded prospectively all patients which were diagnosed of seizure in ED of Hospital Clínico of Zaragoza between November 1th and April $30^{\text {th }}$. Chart review was used to gather definitive diagnosis regarding these patients.

Results: Of the 54,022 patients who presented to the ED during the study period, $137(0.36 \%)$ had complains related to seizures. Sixty one (44\%) of these patients were admitted to the hospital. New-onset seizures were thought to be present in $60 \%$ of patients. Blood work was abnormal in $12 \%$ of patients and $48 \%$ of patients had pathological findings in neuroimaging study. False positive diagnosis was present in $33 \%$ of patients. The most important trigger of seizures in epileptic patients was tapped suddenly the antiepileptic drug treatment.

Conclusions: We found a false positive diagnosis in $33 \%$ of patients, and the most important confounding pathology was sincope and stroke. High percentaje of pathological findings in neuroimaging studies were found. Electroencefalographic and toxicological studies were performed less than is recommended.
\end{abstract}

KEY WORDS: Diagnosis. Epilepsy. Emergency room care. Neurological examination.

Piñol-Ripoll G, Gómez Bitrian J, de la Puerta González-Miró I, Royo Hernández R, Mauri-Llerda JA. Características y manejo de las crisis epilépticas en urgencias y su correlación diagnóstica tras el ingreso. An Med Interna (Madrid) 2008; 25: 168-172.

\section{INTRODUCCIÓN}

Las crisis comiciales representan una de las causas neurológicas más frecuentes de consulta en los servicios de urgencias por detrás de las enfermedades cerebrovasculares (1), representando el $0,3 \%$ de los pacientes que acuden a urgencias (2); y se considera que como mínimo, una de cada 20 per- sonas experimenta una crisis a lo largo de toda su vida (3). Sin embargo, a pesar de su elevada incidencia, persisten dificultades para realizar un correcto diagnóstico cuando el paciente acude a los servicios de Urgencias tras una supuesta crisis, de modo que solo en un 20-40\% de los 27.000 ingresos anuales que se producen en Inglaterra por una crisis comicial se confirma el diagnóstico (4). La falta de pruebas complementarias

Trabajo aceptado: 17 de diciembre de 2007 
específicas hace que la historia clínica y presencia de determinados signos indirectos en la exploración física sean fundamentales para establecer el diagnóstico. Sin embargo, las dificultades para realizar una correcta anamnesis en el Servicio de Urgencias debido, entre otras causas, a la amnesia del episodio o estado postcrítico que suele presentar el paciente, así como las dificultades para aportar una correcta información por parte de los acompañantes; lleva a que en muchas ocasiones no se pueda valorar correctamente a los pacientes con el consiguiente error diagnóstico, de derivación y finalmente de su manejo terapéutico; siendo los síncopes, las crisis psicógenas así como las alteraciones cardíacas y metabólicas las principales causas de error diagnóstico $(5,6)$.

Por este motivo, en este trabajo se registraron todos los pacientes mayores de 14 años que fueron diagnosticados de crisis comicial en el Servicio de Urgencias de nuestro hospital, tanto en pacientes con epilepsia conocida como aquellos sin antecedentes, para valorar sus características demográficas, identificar los distintos servicios donde ingresaron, estancia media hospitalaria en función de los distintos servicios así como la realización de las distintas pruebas complementarias y el rendimiento diagnóstico de las mismas, y finalmente, determinar el porcentaje de pacientes a los que se confirmó el diagnóstico para determinar el grado de eficiencia en el manejo de este grupo de pacientes que suponen un elevado coste sanitario tanto en su manejo crónico como en la fase aguda.

\section{MATERIAL Y MÉTODOS}

Se realizó un estudio descriptivo en el que se incluyeron de forma prospectiva todos los pacientes mayores de 14 años que acudieron al Servicio de Urgencias del Hospital Clínico "Lozano Blesa" de Zaragoza, tanto si requirieron ingreso como no, con el diagnóstico de crisis convulsiva entre los meses de noviembre del 2004 y abril del 2005. Este es el único hospital responsable del área 3 de salud de Aragón que corresponde a una extensión superior a $6.000 \mathrm{~km}^{2}$ compuesto por 116 municipios y un total de 274.533 habitantes, considerando que más del $95 \%$ acuden al servicio de salud público ante una patología potencialmente grave como puede ser una crisis convulsiva.

Las características que se registraron fueron los antecedentes patológicos de los pacientes, características de la historia clínica, servicio al que se derivaron los pacientes, estancia media hospitalaria así como el porcentaje de pacientes a los que se confirmó el diagnóstico de crisis comicial y necesidad de tratamiento en el servicio de urgencias. En cuanto a las exploraciones complementarias se determinó tanto la realización y porcentaje de hallazgos patológicos a nivel analítico, electroencefalográfico (EEG) y en las pruebas de neuroimagen (TC y/o RNM cerebral) tanto si se realizaron en urgencias o posteriormente durante el ingreso hospitalario.

En cuanto al análisis estadístico se ha realizado un estudio descriptivo de las distintas variables estudiadas representándose las variables cuantitativas mediante la media aritmética y desviación estándar, mientras que las variables cualitativas se han caracterizado mediante su distribución de frecuencias. En el análisis de significación se ha utilizado el estadístico de la tstudent para contrastar diferencias entre variables cuantitativas mientras que para el estudio de las variables cualitativas se ha utilizado el estadístico Chi-cuadrado. En todos los valo- res se ha considerado como estadísticamente significativo un valor de $\mathrm{p}<0,05$. Todos los análisis estadísticos han sido realizados con el programa estadístico SPSS (SPSS para Windows, v.13; SPSS, Inc., Chicago, IL).

\section{RESULTADOS}

Durante los seis meses en que duró el estudio 54.022 pacientes fueron atendidos en el Servicio de Urgencias, de los cuales $137(0,25 \%)$ fueron diagnosticados de crisis comicial, requiriendo ingreso hospitalario $61(44 \%)$ de ellos. Durante este mismo periodo de tiempo, el número de ingresos hospitalarios fue de 16.902 pacientes, por lo que las crisis comiciales representaron el 0,36\% del total de los ingresos. Del resto de pacientes, el $41 \%$ fueron dados de alta, permanecieron 24 horas en la sala de observación el $12 \%$ y requirieron ingreso en la Unidad de Cuidados Intensivos el 3\%.

Del conjunto de pacientes ingresados, el $75 \%$ (46) fueron derivados al Servicio de Neurología, lo que representa un $6,5 \%$ de todos los ingresos que se produjeron en dicho servicio (698). En cuanto al resto de servicios a los que ingresaron los pacientes encontramos que el 16\% (10) fueron derivados al servicio de Medicina Interna, 5\% (3) al Servicio de Enfermedades Infecciosas y el restante $4 \%$ a los servicios de Digestivo y Pediatría respectivamente.

En cuanto a las características demográficas de la muestra encontramos que la edad media de los pacientes ingresados fue de $53 \pm 5,5$ años $(51 \pm 4,6$ años para los varones y $55 \pm 7,8$ para las mujeres). Si consideramos las diferencias en cuanto a la edad media en función del servicio en el que ingresaron encontramos que los pacientes ingresados en Neurología tenían una edad media de $51 \pm 6,1$ años, frente a los $37 \pm 10,3$ años de los pacientes del Servicio de Enfermedades Infecciosas o los $67 \pm 4,5$ años de los pacientes ingresados en Medicina Interna.

En cuanto a la distribución por sexos, los hombres representaron el $55,7 \%$ de los ingresos, siendo su prevalencia del $52 \%$ en Neurología y $60 \%$ en Medicina Interna mientras que en el Servicio de Infecciosas el 100\% de los pacientes ingresados por crisis convulsivas durante los 6 meses de estudio fueron varones. El grupo de pacientes que ingresaron en otros servicios tuvieron un $50 \%$ de hombres y mujeres respectivamente.

También se determinó la existencia de factores que potencialmente pueden desencadenar una crisis comicial. Dentro de este grupo se incluyó la existencia de un proceso febril, meningoencefalitis, disminución de las horas de sueño, aumento del stress, consumo o deprivación de alcohol, traumatismo craneoencefálico o dejar de forma brusca los fármacos antiepilépticos, existiendo alguno de estos antecedentes en el $31 \%$ de los casos. En los pacientes con epilepsia el desencadenante más frecuente fue el abandono de la medicación (27\%). La determinación de tóxicos sólo se realizó en el 4,8\% de los pacientes, sin embargo, hasta el 7,3\% de los pacientes en el momento del alta fueron diagnosticados de crisis secundaria al consumo o deprivación enólica y/o consumo de cocaína.

En cuanto a la distribución por meses, y a pesar que los datos se recogieron durante seis meses, observamos que el mes de abril fue el que presentó mayor número de ingresos representando el $25 \%$ del total. El resto de ingresos se distribuyeron de la siguiente manera: noviembre (10\%), diciembre $(13 \%)$, enero $(18 \%)$, febrero $(20 \%)$ y marzo $(14 \%)(\mathrm{p}<0,05)$. 
Encontramos diferencias en cuanto a los días de ingreso hospitalario en función del servicio en el que ingresaron los pacientes. Así, de forma global, la estancia media hospitalaria tras una crisis comicial fue de 10,4 días, siendo de 10,8 para el Servicio de Neurología, 8,4 en Medicina Interna, 13,3 en infecciosas y de 7,7 días en el grupo de otros ( $p<0.05$ ).

$\mathrm{Si}$ atendemos al porcentaje de pacientes que eran epilépticos conocidos, encontramos que el 59\% de los que acudieron a urgencias eran epilépticos conocidos mientras que entre los pacientes que acabaron ingresando este porcentaje representó el $40 \%$. Por servicios, el $45 \%$ de los que ingresaron en neurología era epilépticos conocidos, mientras que en Medicina Interna sólo lo eran el 20 y el 33\% en Infecciosas.

De todos estos pacientes que ingresaron por supuestas crisis convulsivas, se confirmó el diagnóstico en el $67 \%$ (41) de los casos, con un 33\% de errores siendo la causa más frecuente de error: los síncopes (45\%), ictus (21\%), pseudocrisis (18\%) y otros (16\%) (Fig. 1); y se inició tratamiento en urgencias en el $26 \%$ de los pacientes, siendo los fármacos más utilizados las benzodiazepinas.

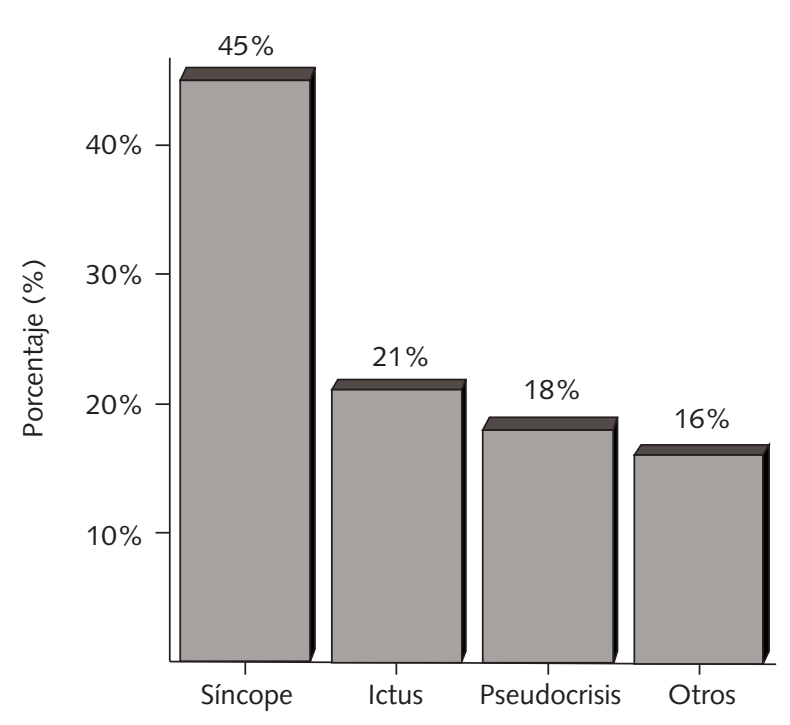

Causas de error diagnóstico

Fig. 1. Representación de las causas más frecuentes de errores diagnósticos en nuestra población de estudio.

Otro aspecto importante del estudio fue evaluar las distintas exploraciones complementarias que se realizan de forma habitual en los pacientes con crisis comiciales (Fig. 2) para poder valorar posteriormente su rendimiento diagnóstico.

En cuanto a los estudios analíticos, el $12 \%$ de los pacientes a los que se confirmó el diagnóstico de una crisis comicial presentaron alteraciones analíticas que podrían justificar las crisis siendo consideradas pues como crisis sintomáticas agudas. El hallazgo más frecuente fueron las alteraciones iónicas, especialmente las alteraciones del calcio y potasio, y en menor frecuencia las alteraciones del sodio.

Entre las pruebas de neuroimagen en los pacientes en que se confirmó la existencia de una crisis epiléptica ésta fue patológica en el $48 \%$ de los casos, siendo la más frecuente la patología vascular (70\%) con una edad media de 73 años, tumoral

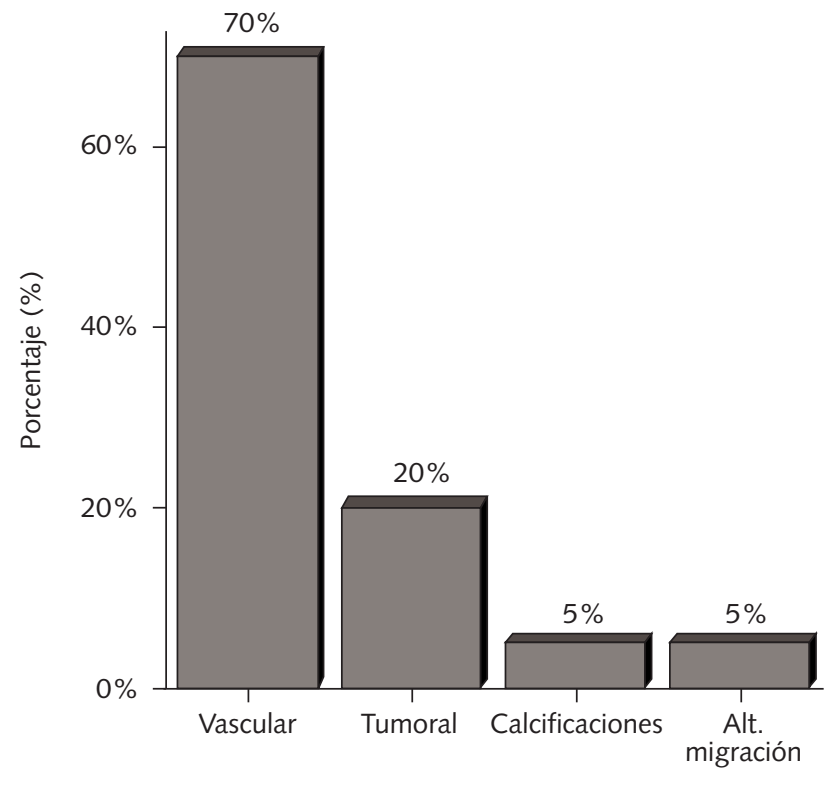

Patología en la neuroimagen

Fig. 2. Hallazgos patológicos en la neuroimagen de los pacientes que acuden a urgencias por crisis comiciales.

(20\%) (61 años) y en menor medida las calcificaciones cerebrales y la displasia cortical focal representando el $5 \%$ de los casos en cada una de ellas y con una edad media de 29 y 22 años respectivamente. Hubo 4 casos en los que no se realizó neuroimagen por ser pacientes epilépticos conocidos sin cambios en las características de sus crisis, por lo que no se estimó necesaria la realización de un nuevo estudio de neuroimagen.

Finalmente, en cuanto a la realización de electroencefalograma, encontramos que sólo a 30 de los 61 pacientes que ingresaron por una supuesta crisis convulsiva se les realizó EEG, lo que supone el $49 \%$ de los pacientes; realizándose solo el $10 \%$ de ellos en urgencias. Los pacientes ingresados en el servicio de neurología fueron a los que se realizó un EEG con mayor frecuencia (54\%) frente al $40 \%$ de los ingresados en Medicina Interna. No se realizó EEG en ninguno de los enfermos ingresados en Infecciosas. En cuanto a su rendimiento encontramos que fue positivo en el $54 \%$ de los pacientes, especialmente cuando el EEG se realizó durante las primeras 24 horas después de la clínica.

Finalmente, otra opción que disponemos para valorar el correcto control terapéutico de los pacientes con crisis ya conocidas es la determinación de los niveles de fármacos antiepilépticos (FAE). Entre los pacientes que ingresaron y que llevaban FAE de los que se pueden determinar los niveles plasmáticos, encontramos que éstos se realizaron en el $46 \%$ de los casos, y de éstos el 33\% tenían niveles bajos o en el rango normal-bajo de las cifras que se consideran eficaces desde el punto de vista terapéutico.

En cuanto a los distintos tipos de crisis encontramos que las más frecuentes fueron las tónico-clónicas generalizadas (47\%), atónicas (13\%), tónicas (9\%), clónicas generalizadas $(5 \%)$, crisis parciales complejas o parciales secundariamente generalizadas $(18 \%)$, crisis parciales simples $(1 \%)$, crisis morfeicas $(1 \%)$, no especificándose el tipo de crisis en el $6 \%$ de los casos. De los pacientes que acabaron siendo diagnosticados de crisis comicial, el $72 \%$ fueron crisis tónico-clónicas, el $2 \%$ eran atónicas, tónicas generalizadas el $7 \%$, clónicas gene- 
ralizadas el $3 \%$, crisis parciales complejas o secundariamente generalizadas el $14 \%$ y crisis morfeicas en el $2 \%$ de los casos.

\section{DISCUSIÓN}

La epilepsia es una enfermedad crónica, sin embargo, mientras el manejo ambulatorio de estas entidades está bien establecido para la mayoría de las enfermedades neurológicas, el impacto sobre el diagnóstico, manejo y tratamiento en la sala de urgencias permanece infraestimado, a pesar que las urgencias neurológicas suponen aproximadamente el $15 \%$ de las consultas de los Servicios de Urgencias (7). Es en éste ámbito donde el papel del neurólogo es crucial en definir, confirmar e implementar el manejo del paciente mediante una adecuada comunicación con los médicos de los servicios de urgencias y médicos de atención primaria para poder ofrecer una asistencia continuada a éste grupo de pacientes evitando errores diagnósticos y de manejo, tal y como se ha demostrado que ocurre en otras enfermedades neurológicas (8). Para ello, los médicos de urgencias, deberían de disponer de la información necesaria para identificar las causas subyacentes de las crisis y decidir si el manejo terapéutico debe ser modificado (9).

En algunos estudios se considera que los falsos positivos y negativos producidos por los equipos de urgencias son del 37,3 y $36,6 \%$ respectivamente en el global de los pacientes neurológicos (1). En el caso concreto de las crisis comiciales, se considera que hasta un $18,7 \%$ de los sujetos tienen un falso positivo diagnóstico, siendo las causas más frecuentes de error la confusión (44\%), síncope $(19 \%)$, ictus y tumores $(11,1 \%)$; mientras que hay un $21,8 \%$ de falsos negativos, siendo también el síncope $(50 \%)$, ictus $(32,9 \%)$ y confusión $(10,5 \%)$ las causas más frecuentes de error diagnóstico $(1,5)$. Estos datos parecen confirmar los resultados de nuestro trabajo, en los que los falsos positivos representan un 33\% de los diagnósticos.

Sin embargo, reconocer un episodio como una crisis puede no ser sencillo y depende especialmente de la historia clínica y en menor medida de la exploración física que tendrían que permitir diagnosticar hasta el $85 \%$ de los pacientes, y solo en pacientes seleccionados, test diagnósticos como bioquímica en sangre, ECG, EEG, TC y monitorización con Holter darían información adicional (10). Sin embargo, en muchas ocasiones es difícil realizar un diagnóstico correcto; pudiendo llevar en ocasiones a infradiagnosticar a los sujetos, o peor aún, empezar el tratamiento antiepiléptico sin tener la confirmación diagnóstica, lo que puede ocasionar efectos sobre el estilo de vida y la autoestima junto con los efectos secundarios de la medicación (11), y en caso de ingreso no orientar correctamente a los pacientes hacia el servicio que deben ingresar.

Para ello la historia clínica debe ser precisa y detallada (preferiblemente confirmada por un observador) y recogida por un médico experimentado, ya que ciertos detalles como los antecedentes de crisis, consumo de alcohol y de drogas, pueden influenciar en el manejo agudo del paciente. Sin embargo, las historias en los servicios de urgencias son limitadas por distintas razones: los pacientes quizás tengan amnesia del evento, presenten confusión post-ictal o están dormidos después de habérseles administrado benzodiazepinas; el acompañante quizás está asustado o no ha presenciado el episodio; y además, el historial clínico previo a menudo no está disponible. Para ello en muchas ocasiones será necesario obtener información adicional ya sea mediante el personal paramédico que pueda describir las crisis en el lugar donde ocurrieron o durante el traslado, o bien, hablando con algún observador o telefoneando a alguna persona cercana (12).

En cuanto al examen físico revelará la causa de las crisis en muy pocas ocasiones, aunque algunos signos indirectos de la exploración puedan ayudar a confirmar la sospecha diagnóstica.

Entre las exploraciones complementarias, las determinaciones sanguíneas de las cifras de glucemia e iones pueden aportar el diagnóstico etiológico de las crisis hasta en un $12 \%$ de los casos como observamos en nuestro trabajo. En casos de epilepsia ya conocida, el nivel plasmático de los fármacos antiepilépticos puede ayudar a confirmar un pobre cumplimiento de los fármacos; así, hasta en un tercio de los pacientes a los que se les realizaron estas determinaciones se encontraron niveles infraterapéuticos. Además, se debe insistir en la necesidad de incrementar el porcentaje de determinación de tóxicos en una sociedad donde el consumo de estas sustancias cada vez es más frecuente. Contrariamente, los niveles de prolactina sérica no suelen estar disponibles en los servicios de urgencias durante las 24 horas por lo que su utilidad se ha mostrado reducida (13).

A diferencia de lo que ocurre con las urgencias pediatricas, donde la realización de pruebas de neuroimagen en ocasiones precede a la propia historia clínica y a la exploración mientras el rendimiento del TC en urgencias es limitado (siempre que no haya un déficit neurológico de instauración de novo o cambios mentales prolongados); porque la mayoría de niños requerirán la realización de una RNM semanas más tarde para completar el estudio etiológico de los síndromes epilépticos que habitualmente debutan en ese periodo de la vida; observamos que existe un elevado rendimiento en la realización de neuroimagen en los pacientes adultos que acuden a los servicios de urgencias por una crisis comicial como demostramos en nuestro trabajo $(14,15)$, siendo pues la técnica de neuroimagen de elección porque permite una estrecha monitorización del paciente durante la prueba en caso que esta sea necesaria, independientemente que de forma programada pueda ser necesaria la realización de una RNM para identificar distintas causas de epilepsia focal como por ejemplo la esclerosis mesial temporal o una displasia cortical, o mejorar el diagnóstico de los hallazgos observados en el TC.

Finalmente, destacamos la importancia de la realización del EEG y que sin embargo permanece infrautilizado, realizándose de urgencia sólo en el 10\% de los casos cuando sabemos que puede ser positivo hasta en el $51 \%$ de los casos cuando se realiza dentro de las primeras 24 horas después de la crisis $(15,16)$. Además, puede permitir distinguir los distintos tipos de crisis de encefalopatias tóxico-metabólicas y psicosis aguda; teniendo una relación coste-eficacia superior a otros tests usados de una forma más amplia como el TC.

En conclusión, observamos que se produce un error en el diagnóstico de las crisis comiciales en urgencias en un tercio de los pacientes, siendo los síncopes y el ictus las causas más frecuentes de error, con el consiguiente error en el manejo y tratamiento de éstos pacientes. Así mismo, observamos un elevado porcentaje de patología en la neuroimagen, mucho mayor del observado en las series pediátricas, lo que demuestra su eficiencia, mientras que insistimos en la necesidad de incrementar la realización de estudios toxicológicos y determinación de los niveles plasmáticos de fármacos en los pacientes que acuden a los servicios de urgencias por una crisis comicial dado que la principal causa de recaída en epilépticos conocidos fue el abandono súbito de la medicación. 


\section{Bibliografía}

1. Moulin T, Sablot D, Vidry E, Belahsen F, Berger E, Lemounaud P, et al. Impact of emergency room neurologists on patient management and outcome. Eur Neurol 2003; 50: 207-14.

2. Craig J, Patterson V, Rocke L, Jamison J. Accident and emergency neurology: time for a reppraisal? Health Trends 1997; 29: 89-91

3. Kotsopoulos IA, Van Medore T, Kessels FG, de Krom MC, Knottnerus JA. Systematic review and meta-analysis of incidence studies of epilepsy and unprovoked seizures. Epilepsia 2002; 43: 1402-9.

4. NHS Hospital Episode Stattistics 2001. p. 77. http://wwwdoh.gov.uk/hes/tables/tb01600a.pdf.

5. Smith PE. If it's not epilepsy... J Neurol Neurosurg Psychiatry 2001; 70 (Supl. 2): 9-14.

6. Delanty N, Vaughan CJ, French JA. Medical causes of seizures. Review. Lancet 1998; 352: 383-90.

7. Adams HP, Brott TG, Crowell RM, Burlan AJ, Gómez CR, Grotta J, et al. Guidelines for the management of patients with acute stroke. A statement for healthcare professionals from a special writing group of the stroke council, American Heart Association. Stroke 1994; 25: 1901-14.

8. Ferro JM, Pinto AN, Falcao I, Rodrigues G, Ferreira J, Falcao F, et al. Diagnosis of stroke by the nonneurologist: A validation study. Stroke 1998; 29: 1106-9.

9. Montouris GD, Jagoda AS. Manegement of breakthrough seizures in the emergency department: continuity of patient care. Curr Med Res Opin 2007; 23: 1583-92.

10. Day SC, Cook EF, Funkenstein H, Goldman L. Evaluation and outcome of emergency room patients with transient loss of consciousness. Am J Med 1982; 73: 15-23

11. Chadwick DW, Smith D. The misdiagnosis of epilepsy. BMJ 2002; 324: 495-6.

12. Smith PE, Cossburn MD. Seizures: Assessment and management in the emergency unit. Clinical Medicine 2004; 4: 118-21.

13. Vukmir RB. Does serum prolactin indicate the presence of seizure in the emergency department patient? J Neurol 2004; 6: 736-9.

14. Freeman JM. Less testing is hended in the emergency room alter a first afebrile seizure. Pediatrics 2007; 3: 194-6.

15. Sharma S, Riviello JJ, Harper MB, Bassin MN. The role of emergent neuroimaging in children with new-onset afebrile seizures. Pediatrics 2003; 111: 1-5.

16. King MA, Newton MR, Jackson GD, Fitt GJ , Mitchell LA, Silvapulle MJ, Berkovic SF. Epileptology of the first-seizure presentation: a clinical, electroencephalographic and magnetic resonance imaging study of 300 consecutive patients. Lancet 1998; 352: 1007-11.

17. Hooshmand $\mathrm{H}$, Maloney M. The role of EEG in the emergency room. Clin Electroencephalogr 1980; 11: 163-8. 